\title{
Computed tomography-guided cervical selective transforaminal epidural block for a patient with bilateral anatomical variations of vertebral artery
}

-a case report-

\author{
Hoon Jung, Jung A Lim, Ki-Bum Park, Seong Wook Hong, Kyung-Hwa Kwak, and Jun-Mo Park \\ Department of Anesthesiology and Pain Medicine, School of Medicine, Kyungpook National University, Daegu, Korea
}

\begin{abstract}
A 56-year-old woman complained of radiating pain to the left arm. She was diagnosed with left-sided foraminal stenosis at the C5-6 level. The neurosurgeon requested a left C6 cervical selective transforaminal epidural block (CSTE). Cervical MRI showed a left-sided large tortuous vertebral artery (VA) at the C5-6 level. Before performing CSTE, a CT angiogram was carried out and showed bilateral tortuous VAs. To minimize adverse events, CSTE was performed with nonparticulated steroids and under CT guidance. Following the procedure, the patient's symptoms were relieved completely. Although complication rates of CSTE are generally low, if it occurs, disastrous situation could be. Additionally, if the patient has anatomical variations, the possibility of a complication occurring is greatly increased. It is therefore important to determine whether the patient has any anatomical variations of the VA before performing procedures such as CSTE, and to ensure that needle placement is correct during the procedure and an appropriate drug, such as a non-particulated steroid, is selected. (Korean J Anesthesiol 2013; 65: 468-472)
\end{abstract}

Key Words: Injections, Steroids, Vertebral artery.

Cervical radicular pain is defined as pain sensed in the upper extremities, caused by irritation of a cervical spinal nerve or its root [1]. It is commonly caused by intervertebral disc herniation or narrowing of the intervertebral foramen, and usually resolves without treatment. However, some patients suffer severely and require treatment.

Conservative treatments, including as bed rest, non-steroidal anti-inflammatory drugs, muscle relaxants, physiotherapy such as traction, and graduated exercise, can be used for cervical radicular pain. If the pain persists despite appropriate use of conservative treatments, cervical transforaminal steroid injection can be a good option [1-3]. Inflammatory reaction of the cervical spinal nerve is thought to be the main cause of cervical radicular pain. A cervical epidural steroid injection can be performed by either an interlaminar or transforaminal approach, and with or without fluoroscopic or computed tomography (CT)

Received: July 9, 2013. Revised: 1st, September 13, 2013; 2nd, October 2, 2013. Accepted: October 2, 2013.

Corresponding author: Jun-Mo Park, M.D., Department of Anesthesiology and Pain Medicine, School of Medicine, Kyungpook National University, 130, Dongdeok-ro, Jung-gu, Daegu 700-721, Korea. Tel: 82-53-420-5863, Fax: 82-53-426-2760, E-mail: pjm4013@naver.com (C)This is an open-access article distributed under the terms of the Creative Commons Attribution Non-Commercial License (http:// creativecommons.org/licenses/by-nc/3.0/), which permits unrestricted non-commercial use, distribution, and reproduction in any medium, provided the original work is properly cited. 
guidance. A transforaminal approach is favored for treating cervical radicular pain [1], as it is believed that this approach allows delivery of the maximum concentration of the medication directly to a suspected pathological site.

Although rare, various complications can be associated with cervical selective transforaminal epidural block (CSTE) [4]. The reported complications are bleeding, seizure, stroke and even death [4-7]. In many cases, these complications occur due to direct vertebral artery (VA) injury or unintentional injection into the VA. If anatomical variations in the VA are present, the possibility of complications may increase greatly.

The authors present a case with bilateral tortuous anatomical variations of the VA that was identified before performing the CSTE, which was considered to have prevented probable adverse events.

\section{Case Report}

A 56-year-old female patient presented with neck and left shoulder pain radiating to the left upper extremity. However, no objective loss of sensory or motor function was observed. Her pain had been aggravated for 10 days. Her visual analogue scale (VAS) score was 7 of 10 . Although conservative treatments, including as resting, traction, non-steroidal anti-inflammatory drug and muscle relaxant were properly done for two weeks, her pain was not improved. The neurosurgeon requested a selective left C6 nerve root block. A cervical spine plain film radiograph showed degenerative cervical spondylosis (Fig. 1A). The radiologist reported that the cervical magnetic resonance image (MRI) revealed a left-sided foraminal stenosis at the C5-6 level. Before performing the procedure, the author re-examined the MRI meticulously. Although the radiologist did not mention the $\mathrm{VA}$, the author detected a tortuous anatomical variation in the VA at the left C5-6 level on the MRI (Fig. 1B). The author ordered a CT angiogram to show the anatomical variations in the VA more precisely. The CT angiogram clearly showed bilateral tortuous anatomical variations in the VA (Fig. 2A). In particular, the $3 \mathrm{D}$ reconstruction image showed a unilateral atypical entrance into the foramen transversarium (FT) of the fifth cervical vertebra and a hypoplastic abnormality of the right VA (Fig. 2B). The diameters of the left and right VA were $4.86 \mathrm{~mm}$ and 2.47 $\mathrm{mm}$ at the lower endplate level of $\mathrm{C} 5$, respectively. The diameter
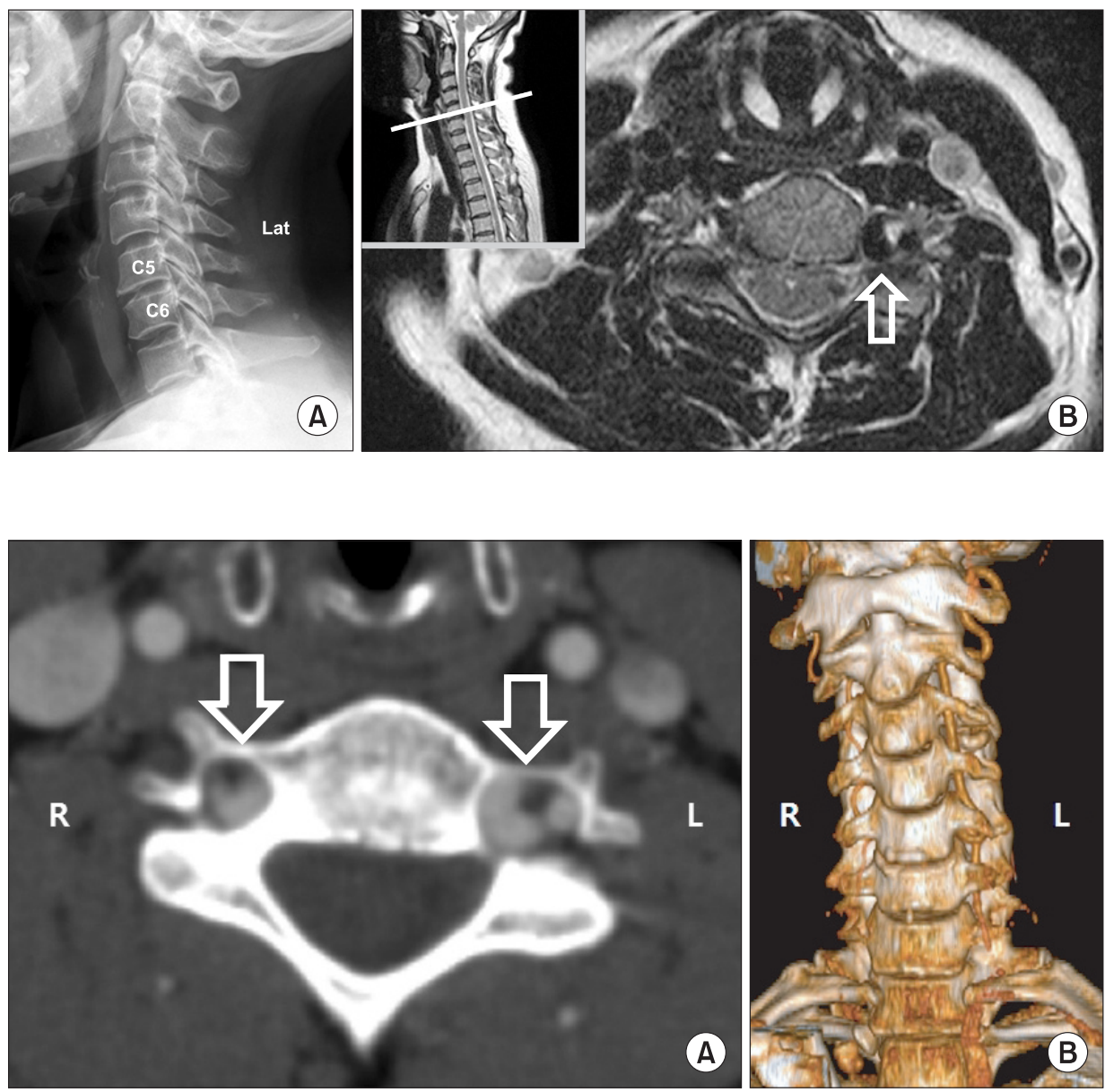

Fig. 2. (A) CT angiogram showing bilateral tortuous anatomical variations in the vertebral artery (VA) (arrows). (B) 3D reconstruction image showing a unilateral atypical entrance into the foramen transversarium of the C5 vertebra and a hypoplastic abnormality of the right VA. 
of the left VA was $2.39 \mathrm{~mm}$ greater than that of the right at the C5 lower endplate. The maximum diameters of the tortuous component of the left and right VA were $9.80 \mathrm{~mm}$ and $6.64 \mathrm{~mm}$, respectively. The maximum diameter of the tortuous component of the left VA was $3.16 \mathrm{~mm}$ greater than that of the right. The left VA entered the FT of the sixth cervical vertebra, whereas right entered the fifth. To minimize adverse events, the procedure was performed with dexamethasone (non-particulated steroid) and under CT guidance. The patient was placed in the supine position on a CT table with her head tilted slightly to the right. Before scanning, a wire was placed on the lateral side of her neck to use as a marker. The patient was scanned to identify a suitable approach point for the $23 \mathrm{G}$ spinal needle. A path that avoided contact with the periosteum and deep cervical plexus was chosen to minimize patient discomfort. The scanning level for the CT fluoroscopy (CTF) image was selected and the target lesion was scanned to a thickness of $1 \mathrm{~mm}$ from the middle of the $\mathrm{C} 5$ body to the middle of the C6 body. The skin entry point and target point for the spinal needle was determined on the CT scan view (Fig. 3A) and marked on the neck. Before the CSTE was performed, $2 \%$ lidocaine was aseptically infiltrated into the skin and subcutaneous tissue. The needle was then advanced toward the target point using intermittent CTF guidance. The needle was advanced slowly into the left C5-6 foraminal area while avoiding the large tortuous VA. It was confirmed that the $23 \mathrm{G}$ spinal needle was located exactly within the left-sided C5-6 foraminal area using $0.3 \mathrm{ml}$ of radio-contrast dye (Fig. 3B). After confirmation of appropriate needle placement, the author injected a mixture of $0.5 \%$ lidocaine $2 \mathrm{ml}$ and dexamethasone 5 mg. Pain was relieved immediately after completion of the procedure and two weeks later, VAS score was decreased to 1 or 0 of 10. The patient's symptoms were relieved almost completely and did not recur in the following 3 months.

\section{Discussion}

CSTE is the injection of local anesthetics and anti-inflamma- tory steroids into the peri-radicular space (the epidural space surrounding the spinal cord and the nerve root) using a transforaminal approach [1]. Despite the safety of this procedure, CSTE carries an inherent risk of serious complications or even death. In many cases, these complications occur due to direct VA injury or unintentional injection into the VA [4-7]. However, some of the complications can be minimized or avoided by promoting awareness of the anatomy or their severity can be reduced by modifying the procedure.

Oga et al. [8] proposed a radiographic grading system for VA tortuosity on anteroposterior cervical spine plain film radiographs and angiograms. They defined the following four types: type 1, the VA is straight and in zone I (outside of the lateral end of the Luschka joint); type 2, the artery is mildly tortuous and in zone I; type 3, the artery has a loop formation, and the most medial portion is in zone II (between the lateral and medial end of the Luschka joint); and type 4, the artery has a loop formation and migration is in zone III (medial to the Luschka joint). In addition, they graded the spondylotic change from a lateral view of the cervical spine radiography as follows: grade 1, no spondylotic change, disc height is normal; grade 2, disc height is low but $>50 \%$ of normal height, and the change is observed in only one level; grade 3, disc height is low at more than two levels or severe narrowing of the disc space $<50 \%$ of normal disc height. They suggested that VA loop formation is associated with cervical spondylotic changes. In the present case, CT angiogram showed a large left VA with a type 3 tortuous anatomical variation according to the grading system of Oga et al. The left VA had a loop and had migrated into zone II. The spondylotic changes on the cervical spine plain radiographs were grade 2 according to the grading system of Oga et al. A hypoplastic VA was at the same time identified on the contralateral side.

Iatrogenic direct VA injuries may be caused by various diagnostic or therapeutic procedures, including diagnostic cerebral angiograms, central venous catheterization, chiropractic manipulation, percutaneous nerve or sympathetic chain block such as a stellate ganglion block (SGB) [5]. They can cause laceration,
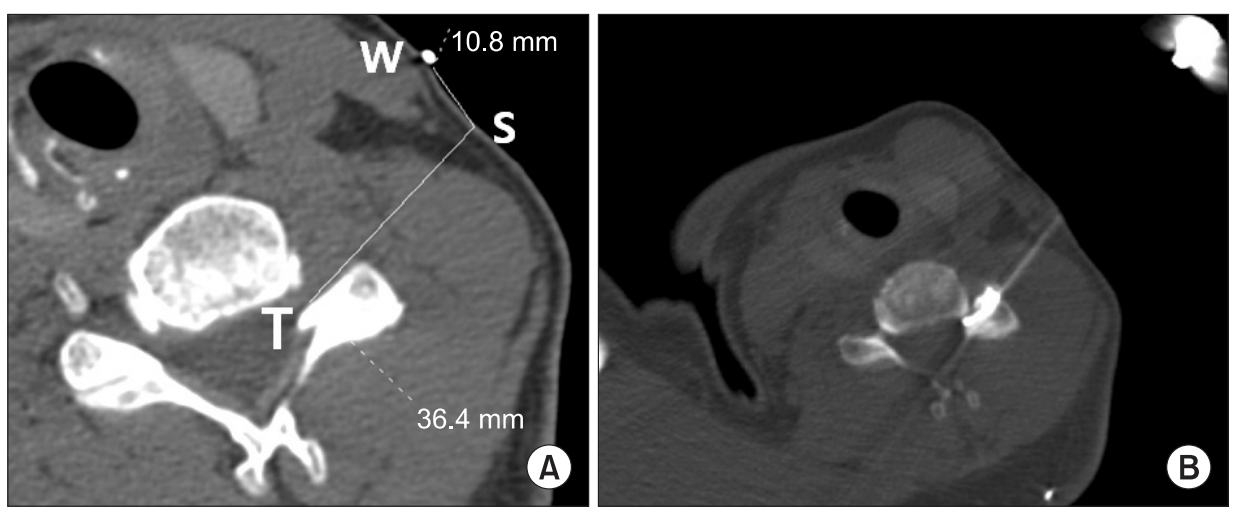

Fig. 3. (A) CT fluoroscopy image showing a wire as a marker (W), skin entry point (S) and target point (T). The distances between $\mathrm{W}$ and $\mathrm{S}$ and between $\mathrm{S}$ and $\mathrm{T}$ were $10.8 \mathrm{~mm}$ and $36.4 \mathrm{~mm}$, respectively. (B) The location of $23 \mathrm{G}$ spinal needle was confirmed by using $0.3 \mathrm{ml}$ of radiocontrast dye. 
arteriovenous fistulae, pseudoaneurysms and arterial dissection of VA. Inamasu and Guiot [5] summarized 16 cases of VArelated complications that occurred during percutaneous nerve block procedures. Most of the reported adverse events resulted not from direct VA injury, but unintentional injection into the VA of local anesthetics or steroids. Only 2 cases of a total 16 cases were the result of direct VA injuries. One was airway obstruction due to laceration and hemorrhage, and the other was brainstem infarction followed by altered mental status due to dissection and thrombosis of VA. The other 14 cases were resulted from injection of local anesthetics or steroids into the VA, which caused reversible grand-mal seizures or transient lockedin syndromes after injection of local anesthetics such as lidocaine or bupivacaine, and a fatal embolic brainstem infarction after particulated steroid injection during CSTE [5,7]. According to Dreyfuss et al. [9], the difference in efficacy between nonparticulated (dexamethasone) and particulated steroids (triamcinolone), was neither statistically nor clinically significant. They suggested that a theoretically safer non-particulated steroids could be a valid alternative to particulated steroids. The author used non-particulated steroid (dexamethasone) in the present case. Half of them occurred during SGBs and just only two cases during cervical nerve root blocks. Although a fatal VA dissection after a transforaminal C7 nerve root block has been reported, it is rare for the arterial puncture itself to cause a major VA injury, because a small-caliber spinal needle is generally used for CSTE to minimize trauma [6]. However, if this did occur, the consequences could be grave. In addition, anatomical variations in the VA can cause disastrous complications and can greatly enhance the occurrence of such complications during various diagnostic or therapeutic procedures. Therefore, recognition of such abnormalities before the procedure is essential [10]. For that reason, CSTE under CT guidance is recommended and was performed in the present case. Although, unlike fluoroscopy guidance, CT guidance may takes longer time, exposes the patient to greater amounts of radiation, and prohibits the dynamic injection of contrast material, it has the advantage of better anatomic resolution and enables direct visualization of the VA [11].

The VA is the main blood supply to the posterior part of the brain [12]. Structures supplied by the VA include the occipital lobe of the cerebrum, the cerebellum, the brain stem, and the upper spinal cord. In the CT angiogram of the present case, the diameter of left VA was larger than the right. Specifically, the diameters of the right VA were $2.47 \mathrm{~mm}$ and $2.33 \mathrm{~mm}$ at the lower and upper endplate levels of C5 and C6, respectively. In a retrospective review, Eskander et al. [13] classified VA anomalies into the following three main groups: intraforaminal, extraforaminal and arterial anomalies. They reported that intraforaminal anomaly refers to midline migration of the VA, which was due to degeneration or trauma in $7.6 \%(19 / 250)$ of patients. In the cases of extraforaminal anomaly, only 92\% (460/500) of VAs entered the FT at the C6 level. In the cases of arterial anomaly, there are also reports of fenestrated, duplicated, and hypoplastic arteries. In Eskander et al's review, hypoplastic VAs were verified in $10 \%(25 / 250)$ of the patients. However, the exact definition and clinical significance of VA hypoplasia has not been established [12,14]. Chen et al. [14] found that a $2.5 \mathrm{~mm}$ VA diameter is ideal for discriminating marked flow asymmetry and low VA flow volume, and proposed a VA diameter $\leq 2.5 \mathrm{~mm}$ to be an ideal definition of VA hypoplasia. It is clear that the present case had hypoplasia of the right VA because the diameter of the right VA was less than $2.5 \mathrm{~mm}$. VA hypoplasia has been generally regarded as a normal variant because the contralateral VA commonly has an increased compensatory flow. However, some authors have recently proposed that VA hypoplasia can be a predisposing factor for posterior circulation stroke [14]. Patients with tortuous VA should understand that the risk of posterior brain infarct is high and we, as health professionals, must explain this to them thoroughly. CT or MRI examination is encouraged for these patients. In many cases, practitioners are wary of injuring the VA during CSTE. Moreover, if unilateral VA hypoplasia is present, as in the present case, injury to the dominant VA may cause lethal complications as a result of insufficient blood flow to the brain stem. Thus, the optimal technique must be carefully selected.

In addition, Eskander et al. [13] reported a spiraling effect that was identified in all patients with migrating VAs. As the VA moves from caudal to cephalad, the VA migrates medially with a clockwise rotation. If interventional treatment (surgery or injections) is planned, this should be considered as very important. And, if this spiraling has a large effect on the nerve root, it may be one of the components of the original pathology. In the present case, radicular pain was thought to be due, not to osteophyte or disc herniation, but to the mass effect of the tortuous VA on the nerve root.

Sometimes, as in the present case, we have found that radiologists do not comment on VA asymmetry when present [12]. If we have any doubts about a radiologist's report, we should examine the scans repeatedly before performing CSTE, to identify whether or not the VA has any anatomical variations.

This is the first report that CSTE was performed for tortuous anatomical variation of VA under CT guidance. Prevention is better than cure. It is important to carefully identify whether any anatomical variations in VA exist before performing procedures, such as CSTE, that can cause vertebral arterial injury, and ensure that needle placement is correct during the procedure and that appropriate drugs, such as non-particulated steroids, are selected. We also recommend that CT guidance (possibly CT fluoroscopy) is used for CSTE if possible, given its significantly greater safety in comparison with fluoroscopy. 


\section{References}

1. Rathmell JP, Aprill C, Bogduk N. Cervical transforaminal injection of steroids. Anesthesiology 2004; 100: 1595-600.

2. Van Zundert J, Harney D, Joosten EA, Durieux ME, Patijn J, Prins MH, et al. The role of the dorsal root ganglion in cervical radicular pain: diagnosis, pathophysiology, and rationale for treatment. Reg Anesth Pain Med 2006; 31: 152-67.

3. Diwan S, Manchikanti L, Benyamin RM, Bryce DA, Geffert S, Hameed H, et al. Effectiveness of cervical epidural injections in the management of chronic neck and upper extremity pain. Pain Physician 2012; 15: E405-34.

4. Wallace MA, Fukui MB, Williams RL, Ku A, Baghai P. Complications of cervical selective nerve root blocks performed with fluoroscopic guidance. AJR Am J Roentgenol 2007; 188: 1218-21.

5. Inamasu J, Guiot BH. Iatrogenic vertebral artery injury. Acta Neurol Scand 2005; 112: 349-57.

6. Rozin L, Rozin R, Koehler SA, Shakir A, Ladham S, Barmada M, et al. Death during transforaminal epidural steroid nerve root block (C7) due to perforation of the left vertebral artery. Am J Forensic Med Pathol 2003; 24: 351-5.

7. Tiso RL, Cutler T, Catania JA, Whalen K. Adverse central nervous system sequelae after selective transforaminal block: the role of corticosteroids. Spine J 2004; 4: 468-74.

8. Oga M, Yuge I, Terada K, Shimizu A, Sugioka Y. Tortuosity of the vertebral artery in patients with cervical spondylotic myelopathy. Risk factor for the vertebral artery injury during anterior cervical decompression. Spine (Phila Pa 1976) 1996; 21: 1085-9.

9. Dreyfuss P, Baker R, Bogduk N. Comparative effectiveness of cervical transforaminal injections with particulate and nonparticulate corticosteroid preparations for cervical radicular pain. Pain Med 2006; 7: 237-42.

10. Eskander MS, Connolly PJ, Eskander JP, Brooks DD. Injury of an aberrant vertebral artery during a routine corpectomy: a case report and literature review. Spinal Cord 2009; 47: 773-5.

11. Wagner AL. CT fluoroscopic-guided cervical nerve root blocks. Am J Neuroradiol 2005; 26: 43-4.

12. Peterson C, Phillips L, Linden A, Hsu W. Vertebral artery hypoplasia: prevalence and reliability of identifying and grading its severity on magnetic resonance imaging scans. J Manipulative Physiol Ther 2010; 33: 207-11.

13. Eskander MS, Drew JM, Aubin ME, Marvin J, Franklin PD, Eck JC, et al. Vertebral artery anatomy: a review of two hundred fifty magnetic resonance imaging scans. Spine (Phila Pa 1976) 2010; 35: 2035-40.

14. Chen YY, Chao AC, Hsu HY, Chung CP, Hu HH. Vertebral artery hypoplasia is associated with a decrease in net vertebral flow volume. Ultrasound Med Biol 2010; 36: 38-43. 